DOUBLY ASYMPTOTIC ORBITS AT THE UNSTABLE EQUILIBRIUM IN THE ELLIPTIC RESTRICTED PROBLEM

\author{
E. Perdios \\ University of Patras, Patras, Greece
}

\title{
ABSTRACT
}

Outgoing asymptotic orbits at a collinear Equilibrium point of the elliptic restricted three-body problem are constructed analytically and numerically and those which intersect perpendicularly the axis of symmetry are selected by means of a numerical procedure of differential corrections. Due to the symmetry properties of the problem, the latter orbits terminate asymptotically back to the equilibrium point and therefore provide a kind of "asymptotic trapping" at the unstable equilibrium, a trapping mechanism based on "long duration of passage" rather than stability of motion.

\section{LINEARIZED EQUATIONS}

The linearized equations of motion of the elliptic planar restricted three-body problem, valid for infinitesimal motion near a collinear equilibrium point, are

$$
\underline{\dot{x}}=\mathbf{A} \underline{\mathbf{x}} .
$$

with

$$
\underline{x}=\left(x_{1}, x_{2}, x_{3}, x_{4}\right)^{T}=(\xi, \eta, \dot{\xi}, \dot{\eta})^{T},
$$

$\xi, \eta$, being the coordinates of the third particle with respect to the equilibrium. The "time"-dependent coefficient matrix $A$ is given by

$$
A=\left[\begin{array}{cccc}
0 & 0 & 1 & 0 \\
0 & 0 & 0 & 1 \\
A^{*} / \sigma & 0 & 0 & 2 \\
0 & B^{*} / \sigma & -2 & 0
\end{array}\right],
$$

where:

V. V. Markellos and Y. Kozai (eds.), Dynamical Trapping and Evolution in the Solar System, 339-350.

- 1983 by D. Reidel Publishing Company. 


$$
\begin{aligned}
& \sigma=1+\epsilon \text { cost, } \\
& A^{*}=1-Q_{0}^{*}-x_{0} Q_{1}^{*}-\mu(1-\mu) R_{1}^{*}, \\
& B^{*}=1-Q_{0}^{*}, \\
& Q_{0}^{*}=\frac{1-\mu}{r_{10}^{3}}+\frac{\mu}{r_{20}^{3}}, \\
& Q_{1}^{*}=-\frac{3}{\alpha r_{10}^{3}}-\mu R_{1}^{*}, \\
& R_{1}^{*}=-3\left[\frac{1}{\alpha r_{10}^{3}}-\frac{1}{(\alpha-1) r_{20}^{3}}\right], \\
& \alpha=x_{0}+\mu, \\
& r_{10}=|\alpha|, r_{20}=|\alpha-1|,
\end{aligned}
$$

We use as "time" $t$, the independent variable $\varphi$ (true anomaly) while, in the above abbreviations, $e$ is the eccentricity of the binary orbit and $x_{0}$ is the position of the equilibrium on the $x$-axis.

2. ANALYTICAL DETERMINATION OF THE OUTGOING EIGENVECTOR

The coefficient matrix $A$ is expanded in the form

$$
A(t)=A_{0}-e A_{1} \cos t+e^{2} A_{1} \cos ^{2} t-\ldots
$$

where:

$$
A_{0}=\left[\begin{array}{llrl}
0 & 0 & 1 & 0 \\
0 & 0 & 0 & 1 \\
A^{*} & 0 & 0 & 2 \\
0 & B^{*} & -2 & 0
\end{array}\right],
$$

and

$$
A_{1}=\left[\begin{array}{llll}
0 & 0 & 0 & 0 \\
0 & 0 & 0 & 0 \\
A^{*} & 0 & 0 & 0 \\
0 & B^{*} & 0 & 0
\end{array}\right] .
$$

The eigen-values of $\mathrm{A}_{0}$ are

$$
\lambda_{1}=\lambda_{0}, \quad \lambda_{2}=-\lambda_{0}, \quad \lambda_{3}=i s_{0}, \lambda_{4}=-i s_{0}
$$


with

$$
\begin{aligned}
& \lambda_{0}=\sqrt{w_{1}+\sqrt{w_{1}^{2}-w_{2}}} \\
& s_{0}=\sqrt{-w_{1}+\sqrt{w_{1}^{2}-w_{2}}}
\end{aligned}
$$

and

$$
w_{1}=(A *+B *-4) / 2, w_{2}=A * B * .
$$

The corresponding eigenvectors are:

$$
\begin{aligned}
& \underline{y}_{1}=\left(1, \gamma_{0}, \lambda_{0}, \gamma_{0} \lambda_{0}\right)^{\mathrm{T}}, \\
& \underline{y}_{2}=\left(1,-\gamma_{0},-\lambda_{0}, \gamma_{0} \lambda_{0}\right)^{\mathrm{T}}, \\
& \underline{y}_{3}=\left(1, i \delta_{0}, i s_{0},-\delta_{0} s_{0}\right)^{\mathrm{T}}, \\
& \underline{y}_{4}=\left(1,-i \delta_{0},-i s_{0},-\delta_{0} s_{0}\right)^{\mathrm{T}},
\end{aligned}
$$

with

$$
\begin{aligned}
& \gamma_{0}=\frac{\lambda_{0}^{2}-A^{*}}{2 \lambda_{0}}=\frac{2 \lambda_{0}}{B^{*}-\lambda_{0}^{2}} \\
& \delta_{0}=\frac{s_{0}^{2}+A^{*}}{2 s_{0}}=\frac{2 s_{0}}{B^{*}+s_{0}^{2}} .
\end{aligned}
$$

In the present case the eigenvalues are single and the eigenvectors are linearly independent, thus

$$
\mathrm{T}_{0}=\left(\underline{y}_{1}, \underline{y}_{2}, \underline{y}_{3}, \underline{y}_{4}\right)^{\mathrm{T}},
$$

is non-singular and ${ }^{A}$ is diagonalized by:

$$
\mathrm{T}_{0}^{-1} \mathrm{~A}_{0} \quad \mathrm{~T}_{0}=\Lambda_{0}
$$

with

$$
\Lambda_{0}=\left[\begin{array}{cccc}
\lambda_{0} & 0 & 0 & 0 \\
0 & -\lambda_{0} & 0 & 0 \\
0 & 0 & i s_{0} & 0 \\
0 & 0 & 0 & -i s_{0}
\end{array}\right] \text {. }
$$


We now put

$$
\mathrm{B}=\mathrm{T}_{0}^{-1} \mathrm{~A}_{1} \mathrm{~T}_{0}
$$

and consider a fundamental solution matrix $\Phi\left(t, t_{0}\right)$ of the system, with $\Phi\left(t_{0} ; t_{0}\right)=I$. Then, we shall have,

$$
\Phi\left(t ; t_{0}\right)=P(t) \quad \exp \left[\left(t-t_{0}\right) L\right]
$$

with

$$
P\left(t+T^{*}\right)=P(t), P\left(t_{0}\right)=I, T^{*}=2 \pi,
$$

I being a constant matrix, whose eigen-vaiues are the characteristic exponents of the problem. Let $T$ be a constant matrix such that

$$
\mathrm{T}^{-1} \mathrm{~L} \mathrm{~T}=\Lambda
$$

where $\Lambda$ is diagonal, and let

$$
\Psi\left(t ; t_{0}\right)=\Phi\left(t ; t_{0}\right) \mathrm{T} .
$$

From (18) and (21) we obtain

$$
\Psi\left(t ; t_{0}\right)=P(t) T \exp \left[\left(t-t_{0}\right) \Lambda\right],
$$

while (21) gives

$$
\dot{\Psi}\left(t ; t_{0}\right)=\dot{\Phi}\left(t ; t_{0}\right) T=A(t) \Phi\left(t ; t_{0}\right) T=A(t) \Psi\left(t ; t_{0}\right),
$$

i.e. $\Psi\left(t ; t_{0}\right)$ is a fundamental solution matrix of system (1).

We now adopt formal series expansions for the above constant matrices $L, \Lambda, T$ as well as for the matrix $Q(t)$, where

$$
Q(t)=P(t) T, Q\left(t_{0}\right)=T .
$$

We shall have:

and

$$
\begin{aligned}
& I=L_{0}+e L_{1}+e^{2} L_{2}+\ldots \\
& \Lambda=\Lambda_{0}+e \Lambda_{1}+e^{2} \Lambda_{2}+\ldots \\
& T=T_{0}+e T_{1}+e^{2} T_{2}+\ldots
\end{aligned}
$$

$$
Q(t)=Q_{0}(t)+e Q_{1}(t)+e^{2} Q_{2}(t)+\cdots
$$


with

$$
Q_{i}\left(t_{0}\right)=T_{i}, \quad i=0,1,2, \ldots
$$

From (22), (23) and (24) we obtain

$$
\dot{Q}(t)=A(t) Q(t)-Q(t) \Lambda
$$

and from (6), (26) and (27) we shall have the following differential Equations accounting for up to fourth-order terms in e:

$$
\begin{aligned}
& \dot{Q}_{0}(t)=A_{0} Q_{0}(t)-Q_{0}(t) \Lambda_{0} \\
& \dot{Q}_{1}(t)=A_{0} Q_{1}(t)-Q_{1}(t) \Lambda_{0} \\
& -A_{1} Q_{0}(t) \cos t-Q_{0}(t) \Lambda_{1} \text {, } \\
& \dot{Q}_{2}(t)=A_{0} Q_{2}(t)-Q_{2}(t) \Lambda_{0} \\
& -A_{1} Q_{1}(t) \cos t-Q_{1}(t) \Lambda_{1} \\
& +\frac{1}{2}\left(A_{1}+A_{1} \cos 2 t\right) Q_{0}(t)-Q_{0}(t) \Lambda_{2} \text {, } \\
& \dot{Q}_{3}(t)=A_{0} Q_{3}(t)-Q_{3}(t) \Lambda_{0} \\
& -A_{1} Q_{2}(t) \text { cost }-Q_{2}(t) \Lambda_{1} \\
& +\frac{1}{2}\left(A_{1}+A_{1} \cos 2 t\right) Q_{1}(t)-Q_{1}(t) \Lambda_{2} \\
& -\frac{1}{4}\left(3 A_{1} \cos t+A_{1} \cos 3 t\right) Q_{0}(t)-Q_{0}(t) \Lambda_{3} \text {, } \\
& \dot{Q}_{4}(t)=A_{0} Q_{4}(t)-Q_{4}(t) \Lambda_{0} \\
& -A_{1} Q_{3}(t) \cos t-Q_{3}(t) \Lambda_{1} \\
& +\frac{1}{2}\left(A_{1}+A_{1} \cos 2 t\right) Q_{2}(t)-Q_{2}(t) \Lambda_{2} \\
& -\frac{1}{4}\left(3 A_{1} \cos t+A_{1} \cos 3 t\right) Q_{1}(t)-Q_{1}(t) \Lambda_{3} \\
& +\frac{1}{8}\left(3 A_{1}+4 A_{1} \cos 2 t+A_{1} \cos 4 t\right) \Omega_{0}(t)-Q_{0}(t) \Lambda_{4} \text {. }
\end{aligned}
$$

We also put

$$
R(t)=T_{0}^{-1} Q(t), \quad R\left(t_{0}\right)=T_{0}^{-1} T
$$

and expand formally, 


$$
R(t)=R_{0}(t)+e R_{1}(t)+e^{2} R_{2}(t)+\cdots
$$

with

$$
R_{i}\left(t_{0}\right)=T_{0}^{-1} T_{i}, \quad i=0,1,2, \ldots
$$

Combining now (15), (17), (26), (28), (29) and (30), we obtain, after some reduction, the following perturbation Equations for the first five terms of expansion (30):

$$
\begin{aligned}
& \dot{\mathrm{R}}_{0}(t)=\left[\Lambda_{0}, \mathrm{R}_{0}(t)\right] \text {, } \\
& \dot{R}_{1}(t)=\left[\Lambda_{0}, R_{1}(t)\right]-B R_{0}(t) \cos t-R_{0}(t) \Lambda_{1} \text {, } \\
& \dot{R}_{2}(t)=\left[\Lambda_{0}, R_{2}(t)\right]-B R_{1}(t) \text { cost }-R_{1}(t) \Lambda_{1} \\
& +B R_{0}(t)\left(\frac{1+\cos 2 t}{2}\right)-R_{0}(t) \Lambda_{2} \text {, } \\
& \dot{R}_{3}(t)=\left[\Lambda_{0}, R_{3}(t)\right]-B R_{2}(t) \cos t-R_{2}(t) \Lambda_{1} \\
& +B R_{1}(t)\left(\frac{1+\cos 2 t}{2}\right)-R_{1}(t) \Lambda_{2} \\
& -R_{0}(t) \Lambda_{3}-B R_{0}(t)\left(\frac{3 \cos t+\cos 3 t}{4}\right) \text {, } \\
& \dot{\mathrm{R}}_{4}(t)=\left[\Lambda_{0}, \mathrm{R}_{4}(t)\right]-B \mathrm{R}_{3}(t) \cos t-\mathrm{R}_{3}(t) \Lambda_{1} \\
& +B R_{2}(t)\left(\frac{1+\cos 2 t}{2}\right)-R_{2}(t) \Lambda_{2} \\
& -R_{1}(t) \Lambda_{3}-B R_{1}(t)\left(\frac{3 \cos t+\cos 3 t}{4}\right) \\
& -R_{0}(t) \Lambda_{4}+B R_{0}(t) \quad\left(\frac{3+4 \cos 2 t+\cos 4 t}{8}\right),(31 e)
\end{aligned}
$$

where we have used the notation

$$
\left[\mathrm{c}_{1}, \mathrm{c}_{2}\right]=\mathrm{c}_{1} \mathrm{c}_{2}-\mathrm{c}_{2} \mathrm{c}_{1}
$$

The solution of the differential Equations (31) finally provides the matrices $R(t)$ and $\Lambda$ to fourth-order terms in e: 


$$
\begin{aligned}
R(t)=I & +\left(C_{11} \dot{\cos t}+s_{11} \sin t\right) e \\
& +\left(C_{20}+C_{22} \cos 2 t+s_{22} \sin 2 t\right) e^{2} \\
& +\left(C_{31} \cos t+s_{31} \sin t+C_{33} \cos 3 t+s_{33} \sin 3 t\right) e^{3} \\
& +\left(C_{40}+C_{42} \cos 2 t+s_{42} \sin 2 t+C_{44} \cos 4 t+\right. \\
\left.\Lambda=\Lambda_{0}+\Lambda_{2} e^{2} \sin 4 t\right) e^{4} & \Lambda_{4} e^{4} .
\end{aligned}
$$

The constant matrices $C_{j j}, S_{j j}, \Lambda_{2}, \Lambda_{4}$ involved in the above solution for $R(t)$ and $\Lambda$ are obtained in the solution process and are given by the following expressions:

$$
\begin{aligned}
& \left(S_{11}\right)_{i j}=-(B)_{i j} /\left[1+\left(\lambda_{i}-\lambda_{j}\right)^{2}\right] \\
& c_{11}=\left[S_{11}, \Lambda_{0}\right] \\
& \left(C_{20}\right)_{i j}=l_{\left[(B)_{i j}-\sum_{k}(B)_{i k}\left(C_{11}\right)_{k j}\right] / 2\left(\lambda_{j}-\lambda_{i}\right), i \neq j} \\
& \left(S_{22}\right)_{i j}=\left\{B\left(I-C_{11}\right)+\frac{1}{2}\left[\Lambda_{0},\left(B S_{11}\right)\right]\right\}_{i j} /\left[4+\left(\lambda_{i}-\lambda_{j}\right)^{2}\right] \\
& \mathrm{C}_{22}=-\frac{1}{2}\left[\Lambda_{0}, \mathrm{~S}_{22}\right]+\frac{1}{4} \mathrm{BS}_{11} \\
& \left(\Lambda_{2}\right)_{i i}=\frac{1}{2}(B)_{i i}-\frac{1}{2} \sum_{k}^{(B)}{ }_{i k}\left(C_{11}\right)_{k i} \\
& \left(S_{31}\right)_{i j}=\left\{\frac{1}{2}\left[\Lambda_{0},\left(B_{22}\right)\right]+\left[\Lambda_{0},\left(S_{11} \Lambda_{2}\right)\right]\right. \\
& -\frac{1}{4}\left[\Lambda_{0},\left(\mathrm{BS}_{11}\right)\right]-\mathrm{B}\left(\mathrm{C}_{20}-\frac{3}{4} \mathrm{C}_{11}+\right. \\
& \left.\left.\frac{1}{2} C_{22}+\frac{3}{4} I\right)-C_{11} \Lambda_{2}\right\}_{i j} /\left[1+\left(\lambda_{i}-\lambda_{j}\right)^{2}\right]
\end{aligned}
$$


346

E. PERDIOS

$$
\begin{aligned}
& c_{31}=-\left[\Lambda_{0}, s_{31}\right]+\frac{1}{2} B\left(s_{22}-\frac{1}{2} s_{11}\right)+s_{11} \Lambda_{2} \\
& \left(S_{33}\right)_{i j}=\left\{\frac{1}{2}\left[\Lambda_{0},\left(B_{22}\right)\right]-\frac{1}{4}\left[\Lambda_{0},\left(B_{11}\right)\right]\right. \\
& \left.-\frac{3}{2} \text { B }\left(C_{22}-\frac{1}{2} C_{11}+\frac{1}{2} I\right)\right\}_{i j} /\left[9+\left(\lambda_{i}-\lambda_{j}\right)^{2}\right] \\
& c_{33}=-\frac{1}{3}\left[\Lambda_{0}, s_{33}\right]+\frac{1}{6} B\left(s_{22}-\frac{1}{2} s_{11}\right)
\end{aligned}
$$

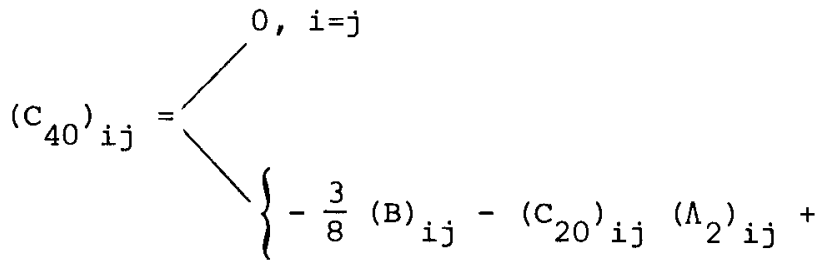

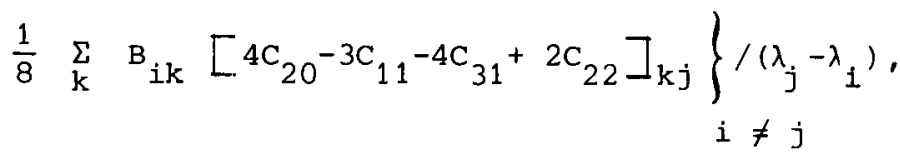

$$
\begin{aligned}
& \left(\mathrm{S}_{42}\right)_{i j}=\left\{\frac{1}{2}\left[\Lambda_{0},\left(\mathrm{BS}_{31}\right)\right]+\frac{1}{2}\left[\Lambda_{0},\left(\mathrm{~B} \mathrm{~S}_{33}\right)\right]\right. \\
& -\frac{1}{2}\left[\Lambda_{0},\left(\mathrm{BS}_{22}\right)\right]+\left[\Lambda_{0},\left(\mathrm{~S}_{22} \Lambda_{2}\right)\right] \\
& +\frac{1}{4}\left[\Lambda_{0},\left(\mathrm{BS}_{11}\right)\right]-\mathrm{B}\left(\mathrm{C}_{31}+\mathrm{C}_{33}-\mathrm{C}_{22}+\mathrm{C}_{20}+\mathrm{C}_{11}-\mathrm{I}\right) \\
& \left.-2 \mathrm{C}_{22} \Lambda_{2}\right\}_{i j} /\left[4+\left(\lambda_{i}-\lambda_{j}\right)^{2}\right] \\
& c_{42}=-\frac{1}{2}\left[\Lambda_{0}, s_{42}\right]+\frac{1}{4} B\left(s_{31}+s_{33}-s_{22}+\frac{1}{2} s_{11}\right)+\frac{1}{2} s_{22} \Lambda_{2} \\
& \left(\mathrm{~S}_{44}\right)_{i j}=\left\{\frac{1}{2}\left[\Lambda_{0},\left(\mathrm{BS}_{33}\right)\right]-\frac{1}{4}\left[\Lambda_{0},\left(\mathrm{BS}_{22}\right)\right]+\frac{1}{8}\left[\Lambda_{0^{\prime}}\left(\mathrm{BS}_{11}\right)\right]\right. \\
& \left.-B\left(2 C_{33}-C_{22}+\frac{1}{2} C_{11}-\frac{1}{2} I\right)\right\}_{i j} /\left[16+\left(\lambda_{i}-\lambda_{j}\right)^{2}\right]
\end{aligned}
$$

https://doi.org/10.1017/S0252921100097220 Published online by Cambridge University Press 


$$
\begin{aligned}
& C_{44}=-\frac{1}{4}\left[\Lambda_{0}, s_{44}\right]+\frac{1}{8} B\left(s_{33}-\frac{1}{2} S_{22}+\frac{1}{4} S_{11}\right) \\
& \left(\Lambda_{4}\right)_{i i}=\frac{3}{8}(B)_{i i}+\frac{1}{8} \sum_{k} B_{i k}\left(4 C_{20}-3 C_{11}-4 C_{31}+2 C_{22}\right)_{k i} .
\end{aligned}
$$

From Equation (22) we now obtain

$$
\Psi\left(t_{0} ; t_{0}\right)=T,
$$

where $\mathrm{T}$ is determined to fourth-order terms in $e$, from the solution process described above. The column of $T$ corresponding to the larger (real) eigenvalue of L-the real positive element of diagonal matrix $\Lambda$ provides the initial state of the outgoing asymptotic orbit leaving the equilibrium. This initial state is normalized by forcing the first component to be equal to 1 , thus introducing the orbital parameter $\varepsilon$ appeaxing in the heading of the table of numerical results below.

The smalness of the orbital parameter $\varepsilon$ is obligatory since we are treating the linearized Equations. An analytical solution, based on a similar perturbation technique, to higher order terms in $\varepsilon$ based on second-or higher-order Equations in the place of Equations (1), is also possible to obtain. In the case of the circular restricted problem $(e=0)$ this has been done by Deprit and Henrard (1965). In the present case of the elliptic restricted problem this perturbation in $\varepsilon$ is much more involved and is not included here. The analysis described here for the collinear equilibria is similar to that of Bennett (1966) referring to the triangular equilibria.

\section{NUMERICAL VERIFICATION}

In practice the initial state of the outgoing asymptotic orbit (for given $\varepsilon$ ) was determined both analytically (as outlined above) and numerically. The numerical determination is based on numerical integration of the linearized system (1). For details on this we refer to Bennett (1965). The results of the two procedures agreed satisfactorily.

\section{SYMMETRIC DOUBLY ASYMPTOTIC ORBITS}

Having obtained the appropriate initial state of the outgoing asymptotic orbit we apply a differential corrections procedure for its "correction" in order to achieve a perpendicular crossing of the axis of the primaries by the orbit. This ensures an asymptotic "return" of the particle back to the unstable equilibrium, at "infinite time" (subject to an additional requirement mentioned below). 
The initial state vector depends on the three parameters :

$$
\mu, e, t_{0}
$$

while the occurrence of a "parpendicular crossing" is equivalent to the satisfaction of the following two conditions:

$$
\begin{aligned}
& y\left(\mu, e, t_{0} ; \tau\right)=0, \\
& \dot{x}\left(\mu, e, t_{0} ; \tau\right)=0,
\end{aligned}
$$

where $\mathrm{x}$ and $\mathrm{y}$ are the usual pulsating - rotating coordinates of the elliptic restricted problem, and $\tau(=n \pi)$ must be an integer multiple of the half-period $\pi$ of the primaries. In the elliptic problem this ensures that the perpendicular crossing is also a "mirror condition" as required for the above mentioned asymptotic return (to the equilibrium), $i$.e for the symmetry of the orbit. $\tau=n \pi$ is therefore the additional requirement mentioned above.

For given value of $\mathrm{n}$ ( $i$.e of $\tau$ ) we have three parameters to adjust in order to satisfy the two conditions (36). This shows that the symmetric doubly asymptotic orbits sought are members of monoparametric sets, and it is easy to set up a predictor-corrector procedure for their numerical determination.

Many doubly asymptotic orbits, belonging to a number of monoparemetric sets-series-of such orbits have been obtained by means of such a procedure and the numerical data corresponding to five such symmetric doubly asymptotic orbits of the elliptic problem (belonging to the same series) and given as examples in the Table below. One of them is shown graphically in the Figure.

Table 1. Symmetric doubly asymptotic orbits $(\varepsilon=-0.001, \tau=6 \pi)$

\begin{tabular}{lllll}
\hline $\mathrm{e}$ & $\mu$ & $t_{0}$ & $\mathrm{x}_{\tau}$ & $\dot{y}_{\tau}$ \\
\hline 0.001 & 0.1782153 & 1.919801 & -2.737897 & 2.249880 \\
0.006 & 0.1810822 & 1.996968 & -2.744719 & 2.257073 \\
0.01 & 0.1834156 & 2.057626 & -2.750137 & 2.262752 \\
0.06 & 0.2149984 & 2.705028 & -2.808980 & 2.321029 \\
0.1 & 0.2403649 & 3.043285 & -2.820386 & 2.322685 \\
\hline
\end{tabular}




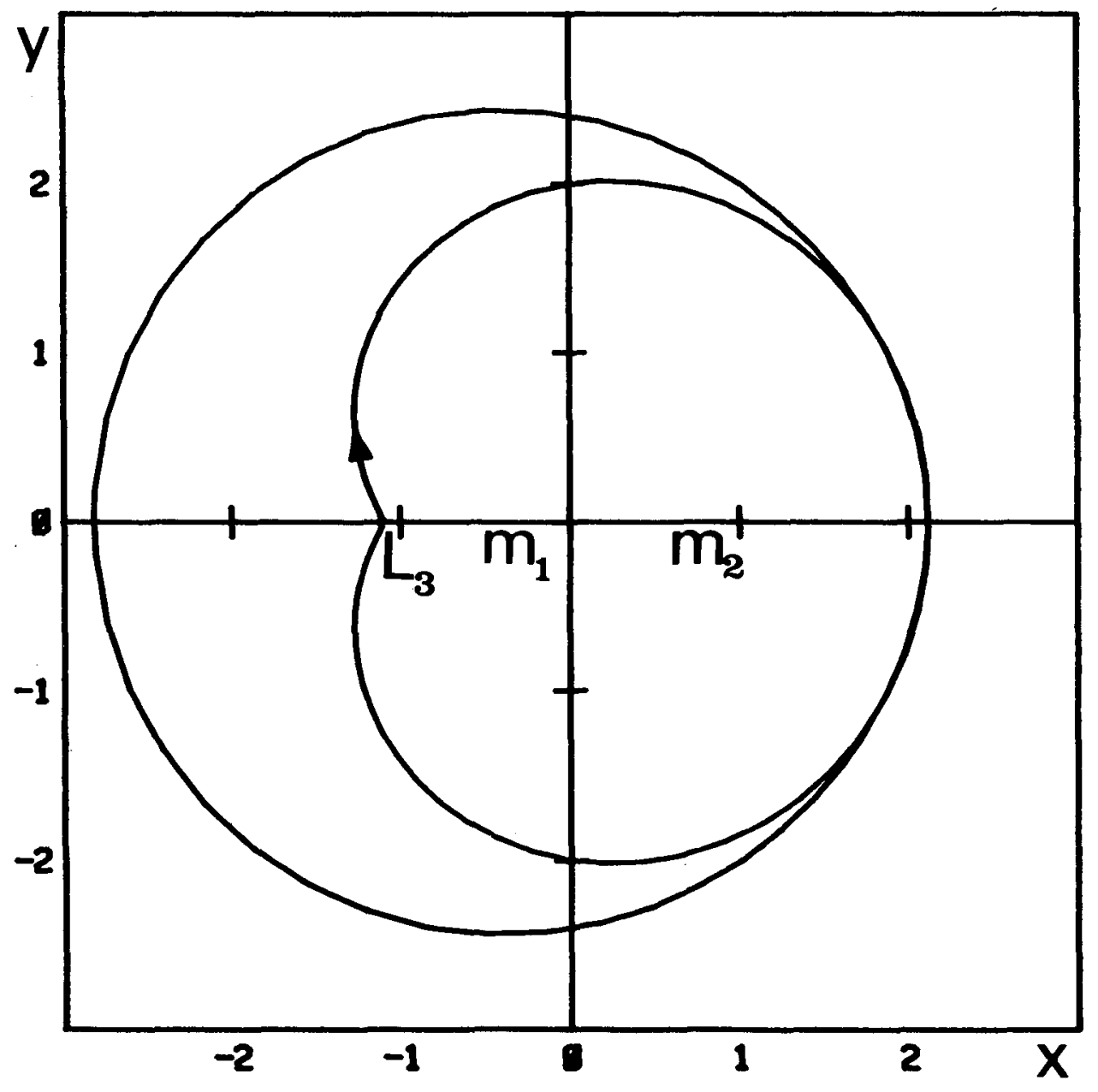

Figure 1. The fifth doubly asymptotic orbit of Table 1.

THE QUESTION OF ASYMPTOTIC TRAPPING

One of the motives behind the present work was to be able to experiment numerically with the idea that an asymptotic orbit in-coming toward the unstable equilibrium can perhaps be considered as representing a trapping mechanism, based on "duration of passage" rather than stability of motion.

With the use of some of the symmetric doubly asymptotic orbits, determined as described earlier, a number of "experimental" numerical integrations "toward the equilibrium" have been performed and the results seem to be encouraging in that, despite the high instability of the integrated orbits, the anavoidable deviations from the model orbits 
seem to result in "long duration of passage", i.e. slow motion near the unstable equilibrium.

\section{ACKNOWLEDGEMENTS}

I wish to thank V.V. Markellos for his help and advice on a number of topics during this work. The analysis of section 2 was done in collaboration with C. Georgiou.

\section{REFERENCES}

Bennett, A.: 1965, Icarus, 4, 177

Bennett, A.: 1966, in R.L. Duncombe and V.G. Szebehely (eds.), Methods in Astrodynamics and Celestial Mechanics, Academic Press, New York, p. 101.

Deprit, A. and Henrard, J.: 1965, Astron. J., 70, 271 